\title{
System Analysis and Design of Gripping Device for Wafer Transfer Robot
}

\author{
Peng Zhang ${ }^{1, ~ a}$, Xianwen Zhuang ${ }^{1, b}$, Lina Hao ${ }^{1, c}$, Hongtao Liu ${ }^{1, d}$ \\ ${ }^{1}$ School of Mechanical Engineering and Automation, Northeastern University, China \\ a z.pyj@163.com, ${ }^{b}$ wonderdsr@163.com, ㄷneuhaolina@163.com, IIht6059198@163.com
}

Keywords: IC manufacture, Wafer transfer robot, End effectors, Edge gripping devices

\begin{abstract}
This paper analyzes the decision method for selecting components of gripping devices for wafer transfer robots from the system point of view and thus provides the basis for type selection and design. Analysis is completed through four steps: First, introduce the role of wafer transfer robot and gripping device in IC manufacture system and analyze the fundamental functions that are essential for wafer gripping; Second, analyze wafer gripping device systematically and divide it into six parts which are called basic elements and provide the implementation methods respectively; Third, analyze and summarize specific requirements to wafer gripping device in wafer transfer process; Finally, analyze and compare the relativity between each basic element and the specific requirement, then give the analysis result in the use of a data table. Through the above analysis of this paper, designers of wafer transfer robot can do analysis and comparison to select suitable components for wafer gripping device, thereby shorten the design circle of robots and improve efficiency.
\end{abstract}

\section{Introduction}

With the rapid development of information industry, IC(Integrated Circuit) manufacture has come into a brand new era and become one of new technologies that contribute to the nation's economy and informationization of the society. IC manufacture equipment plays an important role in IC manufacture, while higher powered IC manufacture equipment is the technical precursor in whole IC industry. The importing of robots satisfies the demand of high efficiency and accuracy. Wafer transfer robots are key facilities of IC manufature equipment which are used to transfer and position wafers between working procedures. The process of wafers consists of several procedures in which robots (Fig. $1^{[3]}$ ) are used to transfer wafers. To avoid wafer dropping during transferring, gripping devices are added to robots' end. There are two types of gripping devices: vacuum and mechanical. Vacuum gripping is to use air pressure to adsorb wafer. Mechanical gripping is to use gripping mechnism to hold wafer's edge, which is called wafer edge gripping device. As wafers are sometimes transferred and processed in a vacuum in which vacuum gripping can not take effect, mechanical gripping devices are used ${ }^{[1]}$. Their performance affects the efficiency and quality directly. Thus, research of wafer transfer robot and wafer gripping device is of positive significance.

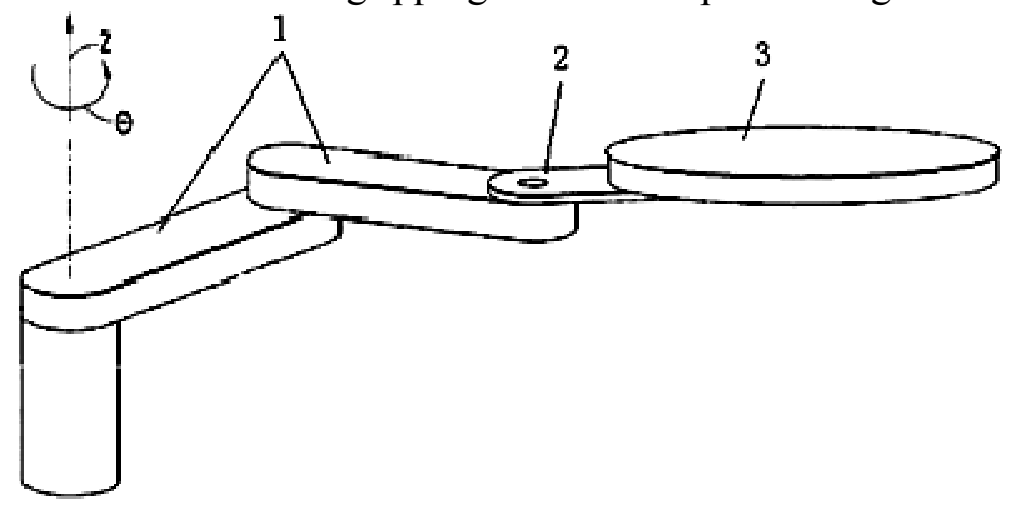

1. robot arms; 2 , end effector; 3. wafer

Fig. 1. Wafer transfer robot 


\section{Functions of wafer edge gripping devices and their implementation}

To transfer wafers accurately with no damage and pollution, wafer edge gripping device should have the following functions: wafer accepting and supporting, wafer introducing, wafer edge contact, wafer fastening and loosening, wafer state detecting and gripping force determining, etc. (Fig. 2). This paper summarizes the implementation of each function and corresponding characteristics. To accomplish system analysis, the functions are called basic elements which affect the performance of gripping devices ${ }^{[2-14]}$.

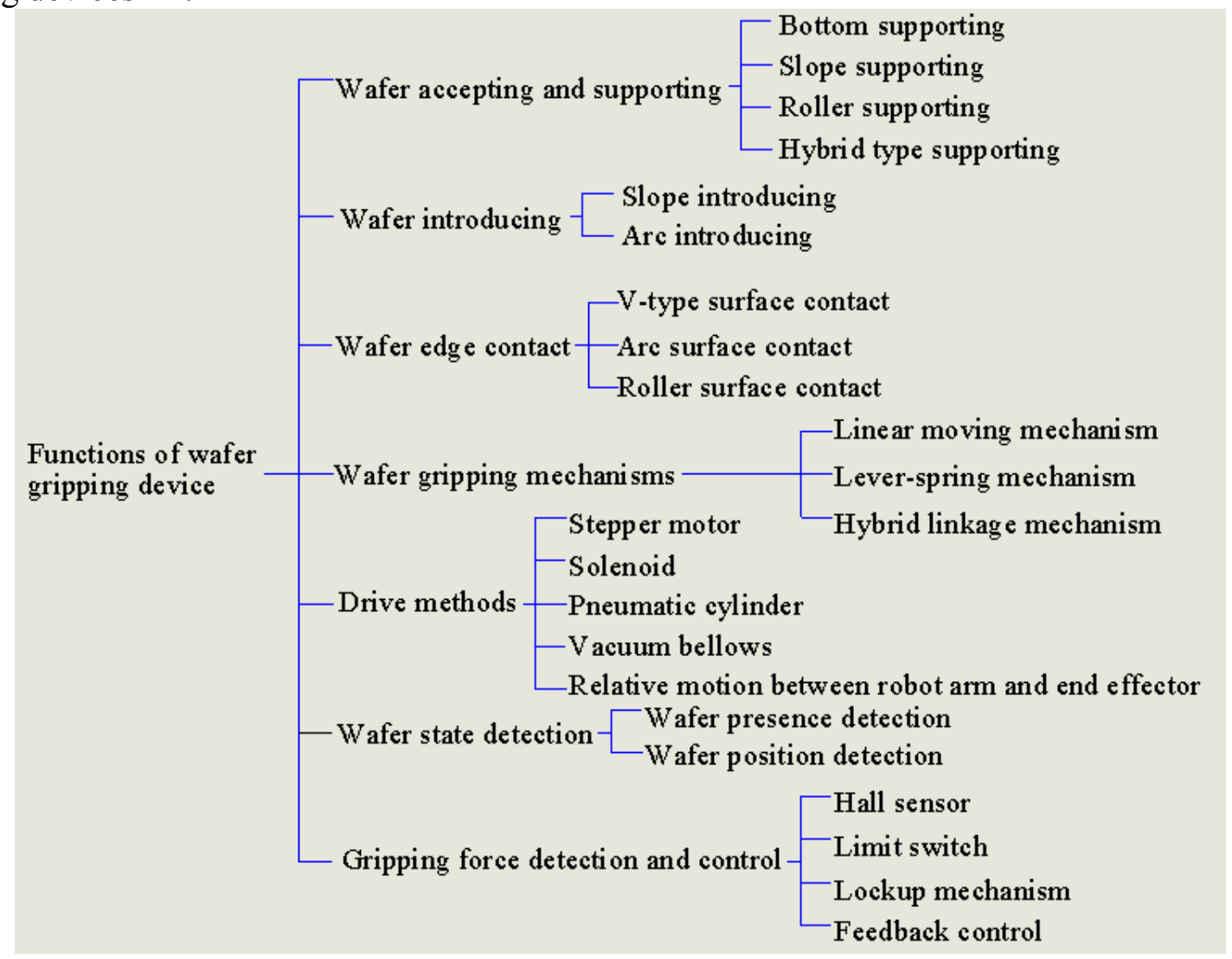

Fig. 2. Functions of wafer gripping device

Wafer accepting and supporting. Before being gripped, wafers are located and supported on robot's end effectors. To avoid damage and pollution during contact, designers should choose local contact method and materials that release fewer particles. Wafer supporting includes bottom supporting, slope supporting, roller supporting and hybrid type supporting. As shown in Fig. $3^{[5]}$, part 2 indicates bottom supporting and part 4 indicates roller supporting. Compared with edge contact supporting, these two types of supporting methods can minimize damage and pollution to the wafer. Further, the contact portion between the wafer and each roller is approximately a point, and it can also minimize friction. Fig. 3 also shows the hybrid type supporting including bottom and roller supporting. Slope supprting (part 2 in Fig. $4^{[6]}$ ) can accomplish edge contact and provide better wafer introducing. The surface of the slope should be smooth enough to minimize friction.

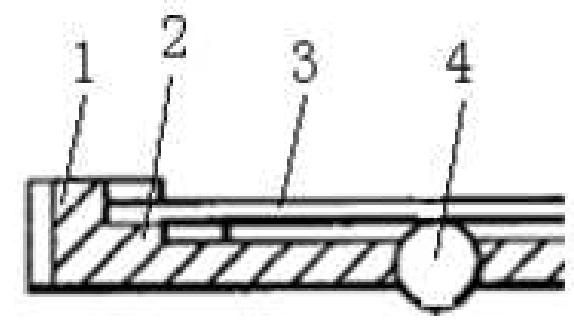

1. end effector; 2. bottom supporting element; 3. wafer; 4. roller

Fig. 3. The combination of bottom supporting and roller supporting 
Wafer introducing. Wafer introducing in one hand can make wafer accepting better, in another hand can provide a transition between pre-gripping state and post-gripping state. Wafer introducing includes: slope introducing and arc introducing. Slope introducing (part 2 in Fig. 4) ensures edge contact of wafers being moved and can minimize damage and pollution. Arc introducing (part 2 in Fig. $5^{[8]}$ ) is similar to slope introducing. It ensures better wafer centering.

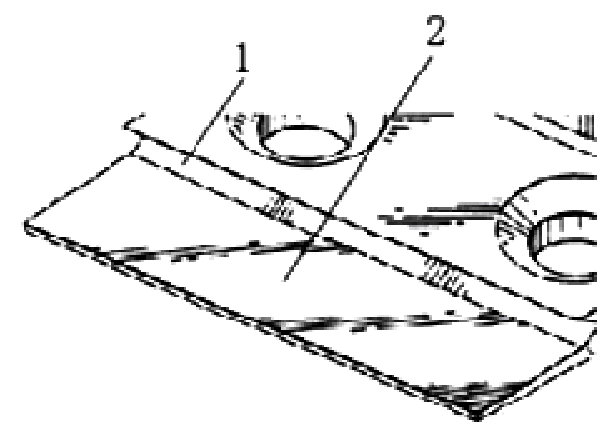

1. contact portion; 2 . introducing portion

Fig. 4. Slope introducing

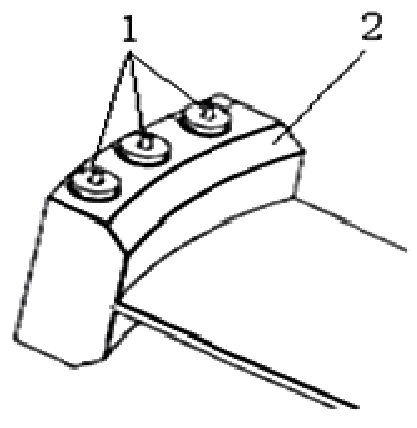

1. rollers; 2. introducing element

Fig. 5. Arc introducing

Wafer edge contact. Wafer gripping is mainly accomplished by contact elements. The shape of contact portion affects contact area, wafer centering and contact force. Wafer edge contact includes: V-type surface contact, arc surface contact and roller surface contact. V-type surface contact can provide stable contact, but if the wafer slants a bit while being pushed by the contact elements, it will stand a force that is perpendicular to the contact surface and points outside the wafer section, which will distort the wafer. Furthermore, if there are more than two V-type contact elements, the restrictions on the wafer will be excessive and make it difficult to center the wafer. (Fig. $6^{[7]}$ ) When the contact element has an arc surface, the force that the wafer stands will point inside the wafer section which will avoid the above problems. (Fig. ${ }^{[7]}$ ) Roller surface contact (part 1 in Fig. 5) is always combined with other contact types to grip wafers. It minimizes the friction between wafer and contact elements. Each contact element should be composed of at least two rollers lest rollers contact with the notch on the wafer.

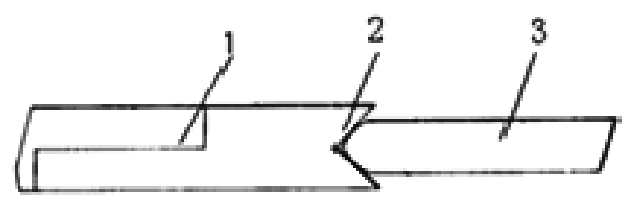

1.end effecior: 2 contact dement; 3 wafe

Fig. 6. V-type surface contact

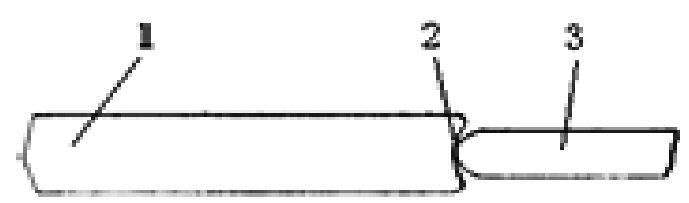

1. end effector, 2 conbact suface; 3 wafer

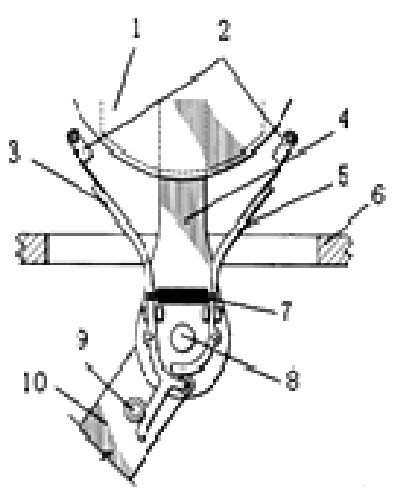

1. wafer 2. contact elements; 3. lever; 4. end effector plate;

5. lever; 6 chamber wall; 7. spring; \&. revolution joint;

9 limit rod; 10. robot am

Fig. 7. Arc surface contact

Fig. 8. Spring-lever mechanism

Wafer gripping (fastening and loosening) mechanisms. There are several mechanisms for wafer gripping, but only the following types are usually used including linear moving mechanism, spring-lever mechanism and hybrid linkage mechanism. Linear moving mechanism is usually composed of a slide rod and a slide. The slide rod can move linearly in the slide. One end of the slide rod is connected to a driver and the other end is connected to the contact elements. Thus the linear 
movement of the contact element is accomplished. Spring- lever mechanism is shown by Fig. $8^{[11]}$, there are contact elements at one end of the two levers which are forced to clamp by the spring. When the wafer either comes or leaves, the other end of one lever is activated by a driver to open the 'clamp'. And hybrid linkage mechanism is shown in Fig. $9^{[12]}$, the mechanism is composed of the slide rod's linear movement and the lever's rotation. It makes gripping more flexible like fingers. Furthermore, this kind of mechanism can also achieve better wafer centering.

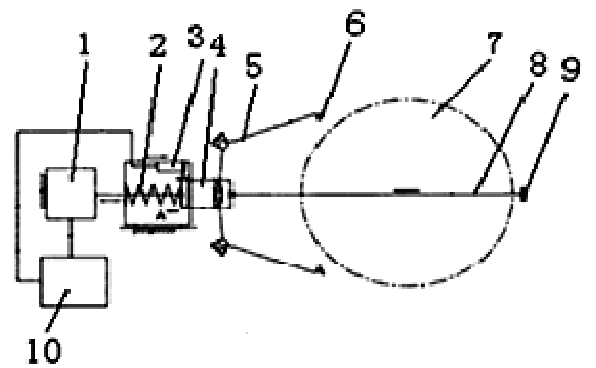

1. driver; 2. spring; 3. sensor; 4, connecting element;

5. lever, 6. contact element; 7. wafer; 8. leader;

9. contart element; 10. controller

Fig. 9. Hybrid linkage mechanism

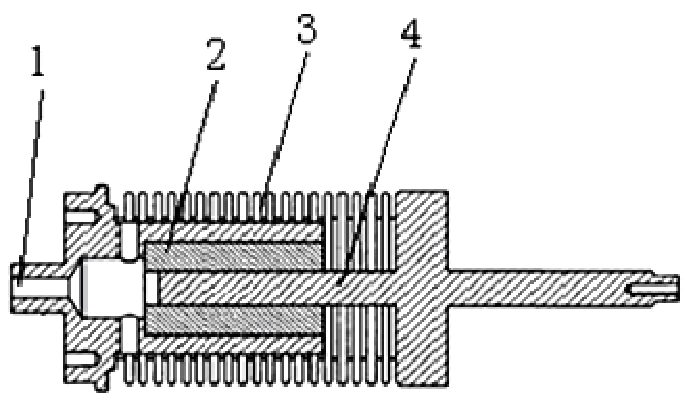

1. air inlet; 2 . bearing; 3 . bellows; 4 . piston rod

Fig. 10. The inner structure of vacuum bellows

Drive methods. To accomplish wafer gripping, linear drivers are installed to robot end effectors. Drivers that are frequently-used include: stepper motor, solenoid, pneumatic cylinder, vacuum bellows and relative motion between robot arm and end effector. Stepper motor can convert input pulse signal into linear or revolution displacement. It achieves accurate motion control but its efficiency is low. Stepper motor may also lose steps and produce oscillation. A solenoid is a device which converts energy into linear motion. This energy comes from an electromagnetic field. By changing the electromagnetic field, it can achieve motion control. Pneumatic cylinders can convert the energy of compressed air into linear motion but can't achieve accurate motion control. Fig. 10 ${ }^{\text {[13] }}$ shows the inner structure of vacuum bellows. It's an extendible bellows connected to vacuum device. When evacuated, the vacuum bellows is compressed. When the bellows is filled with air, it will extend. Thus smooth linear movement is achieved. The structure of vacuum bellows is simple and easy to achieve. Besides the above drive methods, relative motion between robot arm and end effector is also used. (Fig.8) When the angle between robot arm and the gripping device reaches a predetermined value as the robot arm extends towards the wafer storage device, the gripping device will loosen the wafer. Contrarily, when robot arm is retracted from the storage device, the gripping device will fasten the wafer.

Wafer state detection. Wafer state detection includes wafer presence detection and wafer position detection. Wafer presence indicates presence in wafer storage devices or on gripping devices. The detection is usually finished by thru beam sensor which is composed of laser emitter and laser receiver. (Fig. 11 ${ }^{[13]}$, Fig. 12 $2^{[3]}$ ) Wafer position detection indicates determining the displacement between wafer center and a certain position on the gripping device. It's difficult to detect wafer center directly, then we detect the gripping device instead. For example, we can detect the position of a certain point on the contact element.

Gripping force detection and control. In order to grip wafers stably and not to damage them, the gripping force should be adequate (0.01-3kgf) and invariable. Generally, gripping force detection and control are accomplished by Hall sensor, limit switch, lockup mechanism or the combination of the above. Hall sensor is used to determine the relative displacement between a certain point on gripping device and a reference point. Thereby we can determine the gripping force indirectly. When the contact element of the gripping device arrives at a predetermined position, it will touch the limit switch. Then the motion will stop in order to limit the gripping force. And when the gripping force reaches an ideal value, the lockup mechanism can hold the gripping device to keep the gripping force invariable. By determining the gripping force directly using force sensors, the robot can send orders to the driver to do feedback control of the gripping force. 


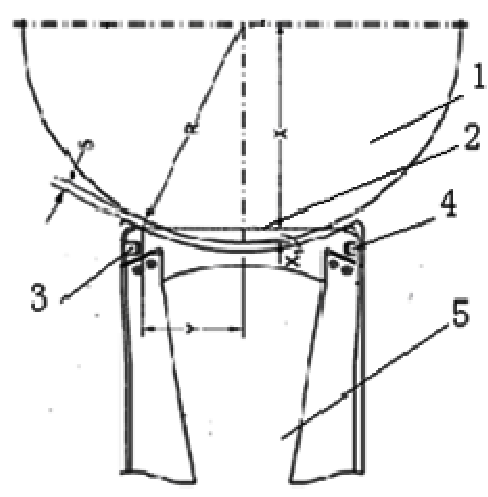

1.wafer, 2. light be am; 3. laser emitter;

4. laser reciever, 5 . end effector

Fig. 11. Wafer presence detection in wafer storage devices

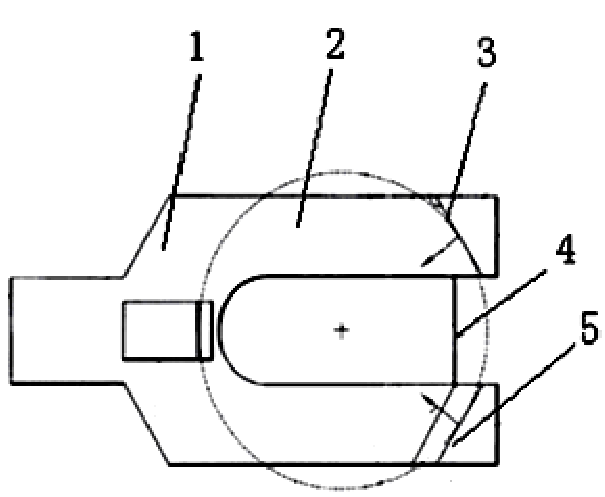

1. and effector, 2. wafer, 3, laser emitter;

4. light beam; 5 .laser recievert

Fig. 12. Wafer presence detection on wafer gripping devices

Application example. Fig. 13 and Fig 14 show one application of gripping device. The end effector is used for transferring and aligning a wafer to be moved, for example, from plasma etch station in a cluster tool to a deposition station or from a manufacturing station to a testing station. The alignment is accomplished by detecting the notch (part 5 in Fig. 14) using notch detector (part 7 in Fig. 13) and by rotating the wafer using roller 6 (Fig. 13) activated by a stepper motor (not shown) ${ }^{[14]}$. Now we can analyze the gripping device using the knowledge from last section:

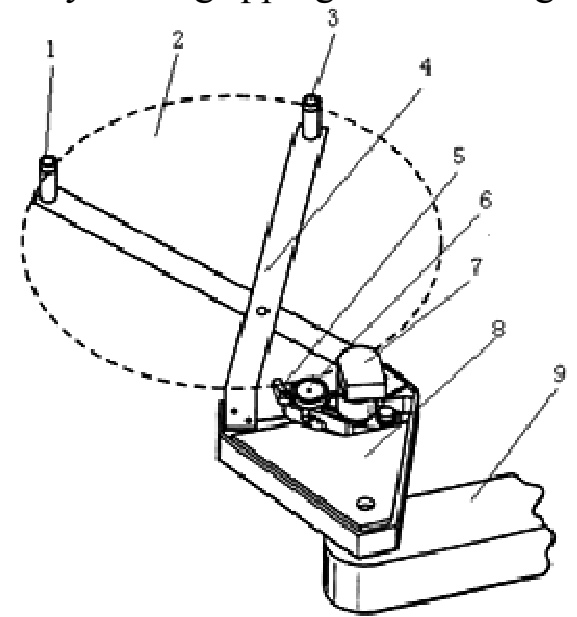

1. roller; 2. wafer; 3. roller; 4. linkage mechanism;

5. supporting element; 6. roller; 7, notch detector;

S. end effector: 9. robot arm

Fig. 13. End effector with notch detector

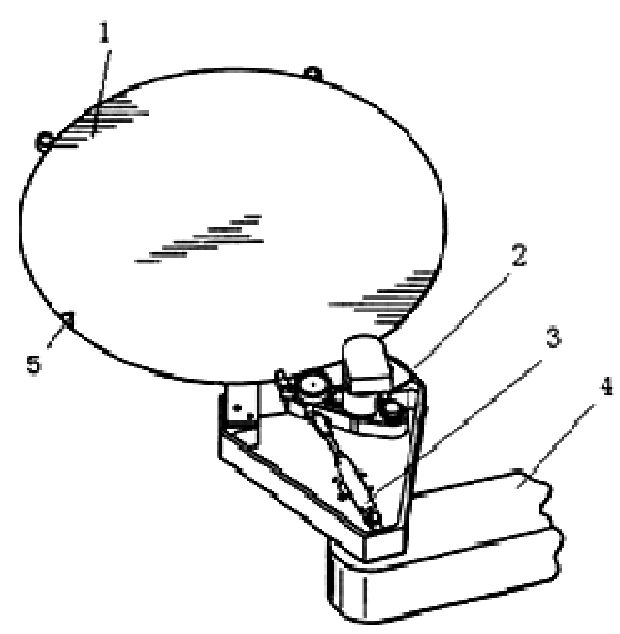

1. wafer ; 2 end effector; 3 . linear driver;

4. robot arm. 5. noteh

Fig. 14. Drive method of the end effector

Wafer supporting: bottom supporting (parts 1, 3, 6 in Fig. 13); Wafer introducing: slope introducing (part 5 in Fig. 13); Wafer edge contact: roller surface contact (parts 1, 3, 6 in Fig. 13);

Wafer gripping mechanism: linear moving mechanism (Fig. 14); Drive methods: rotational driver (stepper motor) for roller 6 (Fig. 13) and linear driver (part 3 in Fig. 14) for gripping device.

With the above functions, this end effector can achieve advantanges of little pollution to the wafer, small friction, better wafer centering and stable gripping. However, as there is only one roller at each contact position, the rollers may contact with the notch. This will affect the detection of the notch and wafer centering.

\section{Relationship between specific requirement and fundamental elements}

To transfer wafers accurately with little damage and pollution, designers should analyze the requirement of each part in more detail, including: mass, center of mass, inertia, dimension, velocity, acceleration, strength, gripping force, and wafer centering. The changes of each part of the gripping 
device lead to the change of the wafer transfer process. So we should analyze the relationship between specific requirement and fundamental elements. According to Table 1 for instance, ' 1 ' indicates that the specific requirement will affect the funamental element, and ' 0 ' means not ${ }^{[15]}$.

Table 1 The relationship between specific requirement and fundamental elements

\begin{tabular}{|c|c|c|c|c|c|}
\hline element & $\begin{array}{c}\text { contact } \\
\text { element }\end{array}$ & $\begin{array}{c}\text { gripping } \\
\text { mechanism }\end{array}$ & $\begin{array}{c}\text { drive } \\
\text { method }\end{array}$ & $\begin{array}{c}\text { detection } \\
\text { device }\end{array}$ & $\begin{array}{c}\text { control } \\
\text { system }\end{array}$ \\
\hline mass & 1 & 1 & 1 & 0 & 0 \\
\hline center of mass & 1 & 1 & 1 & 0 & 0 \\
\hline inertia & 1 & 1 & 1 & 0 & 0 \\
\hline dimension & 1 & 1 & 1 & 0 & 0 \\
\hline velocity & 1 & 1 & 1 & 0 & 0 \\
\hline acceleration & 1 & 1 & 1 & 0 & 0 \\
\hline strength & 1 & 1 & 1 & 0 & 0 \\
\hline gripping force & 1 & 1 & 1 & 1 & 1 \\
\hline $\begin{array}{c}\text { wafer } \\
\text { centering }\end{array}$ & 1 & 1 & 1 & 1 & 1 \\
\hline
\end{tabular}

\section{Conclusion}

This paper analyzes wafer transfer process systematically. We summarize functions that gripping devices should have and specific requirement in wafer transfer process. Furthermore, we analyze the relativity between each implementation method of the basic elements and the specific requirement, the analysis result of which is shown in a data table.

\section{References}

[1] Shen Baohong. Research and Design of Clean Robot Oriented to IC Manufacturing [D].Dalian: Dalian University of Technology, 2006.

[2] Martin Hosek, Timothy Bleigh. Brooks Automation expands control capability of precision industrial robots[J]. Industrial Robot, 2002, 29(4):334-338.

[3] DiBella ta al. Inertial wafer centering end effector and transport apparatus [P]. US Patent: 2008267747A1. 2008-10-30.

[4] Asano. Wafer holding [P].US Patent: 2004048474A1. 2004-3-11.

[5] Ebbing et al. Substrate clamping apparatus[P]. US patent: 6155773. 2000-12-5.

[6] Mankanme et al. Edge gripping end effector wafer handling apparatus[P]. US Patent: 6491330. 2002-12-10.

[7] SINHA et al. Wafer Gripping Fingers[P]. WO patent: 0054941. 2000-9-21.

[8] Gonzalez. Dual wafer edge gripping end effector and method therefore[P]. US patent: 20020071756.

[9] Yokota et al. Robot arm edge gripping device for handling substrates[P]. US Patent: 20030133780. 2003-7-17.

[10]Rice et al. Substrate gripper for a substrate handling robot[P]. US Patent: 7374391. 2008-5-20.

[11]Sundar. Mechanical gripper for wafer handling robots[P]. US Patent: 6322312. 2001-11-27.

[12]Kesil et al. Soft-touch gripping mechanism for flat objects[P]. US Patent: 2004102858A1. 2004-5-27.

[13]Florindi et al. $200 \mathrm{~mm}$ notched/flatted wafer edge gripping end effector[P]. US Patent: 20050110287. 2005-5-26.

[14]Preston Whitcomb. Edge-gripping pre-aligner[P]. US Patent: 6468022B1. 2002-10-22.

[15]Zhang Yuanqi, Zhang Guoxing.System reaserch of robot end effectors [J].Mechanical Manufacture,1997 (9):9-11. 